\title{
An Attempt to Explore various factors involved in the Treatment of Strabismus Eye Surgery and pre as well as Post-surgery Optometric Eye Care
}

\author{
Lokik Aggarwal \\ Recventures Education Services \\ Private Limited \\ Delhi, India
}

\author{
Remica Aggarwal \\ MIT School of Education \& \\ Research, MIT Art \\ Design \& Technological University \\ Pune, India
}

\author{
V. K. Aggarwal \\ Recventures Education Services \\ Private Limited \\ Delhi, India
}

\begin{abstract}
Eyes are the indispensable part of the body. An optometrist is a health care professional who specializes in function and disorders of the eye, detection of eye disease and some types of eye disease management. An Ophthalmologist often face the challenges of treating different categories of patients Present research article focuses on exploring the field of strabismus eye surgery and thereby diagnosing various causes of its occurrence and its treatments .
\end{abstract}

\section{Keywords}

Neuro- Ophthalmology ; Strabismus eye surgery ; Optometry

\section{INTRODUCTION}

Neuro-Ophthalmology is a super specialty that merges the fields of neurology and ophthalmology. Neuroophthalmologists are responsible for the diagnosis and management of complex systemic diseases of the nervous system that affect vision, eye movements and alignment, as well as pupillary reflexes. Often, the symptoms that prompt such a referral include those associated with optic nerve disease or diseases of the visual pathway (the nervous system component that connects the eyes to the brain). Other reasons could be the diseases affecting the pupils of the eye, and certain kinds of squint (especially paralytic). The typical symptoms that could signify a neuro-ophthalmological problem includes sudden decrease or loss of vision; sudden transient loss of vision (called transient ischemic attack or eye stroke); visual hallucinations ; double vision or diplopia ; Intractable headaches; pupillary abnormalities (sluggish reaction, the difference in size of the pupils); sudden onset of difficulties in identifying colors; inability to tolerate bright light; visual field defects and squint or strabismus (especially adult onset)

Following are the common eye diseases that require attention from a neuro-ophthalmologist:

Optic Neuritis: This disease is often associated with Multiple Sclerosis (MS). It is a condition which presents as a sudden onset loss or decrease in vision due to inflammation of the optic nerve.

Papilledema: Papilledema is characterized by the swelling of the optic nerve head due to increased pressure from inside the brain. It may be due to tumors, infections like meningitis, encephalitis, etc.

Toxic or Nutritional Optic Neuropathy: The optic nerve may be damaged due to toxic substances found in tobacco \& alcohol.
Squint or Strabismus : A misalignment of the eye, especially when sudden in onset, and associated with double vision is often due to paralysis of one or more of the small muscles of the eye, and is called paralytic strabismus. The eye shows limitations of ocular movement as well.

\subsection{On various eye care professions [1-4]}

1.1.1 Ophthalmology and Ophthalmologist: Ophthalmology is a branch of medicine that specializes in the anatomy, function, and diseases of the eye. An ophthalmologist diagnoses and treats refractive, medical and surgical problems related to eye diseases and disorders. They have the initials as "M.D." (Doctor of Medicine) or "D.O." (Doctor of Osteopathy) after his or her name. Ophthalmologists are trained to provide the full spectrum of eye care, from prescribing glasses and contact lenses to complex and delicate eye surgery. Ophthalmologists treat eye diseases, prescribe medications, and perform all types of surgery to improve, or prevent the worsening of eye and vision-related conditions.

1.1.2 Optometry and Optometrists : Optometry is a vision care specialty that is concerned with the health of the eyes, the visual system, and related structures. An optometrist is a health care professional who specializes in function and disorders of the eye, detection of eye disease, and some types of eye disease management. An optometrist conducts eye examinations, prescribes corrective contact lenses and glasses, and diagnoses and treats eye diseases and disorders. An optometrist will have the initials "O.D." (Doctor of Optometry) after his or her name.

1.1.3 Orthoptist : An Orthoptist is a certified allied health professional who works under the supervision of an ophthalmologist to evaluate and treat disorders of the visual system with an emphasis on binocular vision (using both eyes to see) and eye movement problems. Orthoptists most commonly work in pediatric ophthalmology settings.

1.1.4 Optician : An Optician is a health professional who is trained to supply, prepare, and dispense optical appliances through interpretation of written prescriptions. An optician fits and finishes eyeglass lenses and frames and may also dispense low vision devices, contact lenses, and artificial eyes. Opticians typically learn job skills through formal on-the-job programs. This training includes technical instruction in measuring eyes or adjusting frames under the supervision of an experienced optician. 


\subsection{Case study Squint or Strabismus}

Strabismus or squint is a misalignment of the eyes where the two eyes are pointed in different directions. Though it is a common condition which affects children, it may appear later in life. Strabismus is usually caused by misaligned eye muscles. However, the exact reason for the misalignment of the eyes leading to Strabismus is not fully understood. Many factors can be responsible for Strabismus. These may include:

(i) Inappropriate development of the "fusion center" of the brain, problems with the "eye movement" centers of the brain, and injury to or disease of the eye muscles or nerves. This explains why children with cerebral palsy, Down's syndrome and hydrocephalus often have Strabismus.

(ii) Even a brain tumor may cause Strabismus.

(iii) Another factor is Genetics, and it is known that Strabismus may run in families. However, in many patients there are no relatives with the problem. The condition occurs equally in males and females.

(iv) Associated eye conditions may also give rise to Strabismus. In cases of cataract, injury or tumor within the eye, the eye may frequently turn in or out.

(v) Certain disorders such as Thyroid disease ; after cataract surgery ; after retinal detachment surgery; Myasthenia Gravis ; paralysis of eye muscles due to diabetes and hypertension and Orbital fracture could be certain disorders which are associated with Strabismus.

Symptoms of the problem : The primary symptom of Strabismus is an eye that is not straight. The misalignment could be permanent and always noticeable (which is known as Constant Strabismus), or it may come and go, appearing normal at times and abnormal at others (which is known as Intermittent Strabismus). One eye may be directed straight ahead while the other eye is turned inward, outward, upward, or downward. In other cases, the turned eye may straighten at times, and the straight eye may turn (alternating strabismus). Sometimes a youngster will close one eye in bright sunlight. Faulty depth perception may be present.

Treatment of the problem : The goals of treatment are to preserve vision, straighten the eyes, and restore binocular vision. Different ways of treating the problem depends upon its level of severity

(i) Treatment for strabismus may be non-surgical and include eye drops or glasses.

(ii) If surgical treatment is indicated, it is wise to align the eyes when the child is young in order to allow more normal use of the eyes together.

(iii) Treatment of Strabismus depends upon the exact cause of the misaligned eyes. It can be directed towards unbalanced muscles, cataract removal or other conditions that are causing the eyes to turn.

(iv) Covering or patching the good eye to force use of the amblyopic eye may be necessary to ensure equal vision. Constant Strabismus must be dealt with immediately if one wants to re-establish proper use of the eyes. Treatment for this condition needs to be early and aggressive. If the eye turn is constant and simple things like patching, glasses (bifocal, prismatic, etc.) do not eliminate the eye turn, surgery needs to be considered. With intermittent Strabismus, the eye does not turn in all the time, so the brain is probably receiving appropriate stimulation for the development of binocular vision. Children with intermittent eye turns should be handled with judicious patching, special glasses, and/or orthoptics (special eye exercises designed to encourage binocular vision). Surgery, if considered at all, should be a last resort. The two most common types of strabismus are esotropia, where an eye turns in and exotropia, where an eye turns out.

\subsubsection{Strabismus signs and symptoms}

The primary sign of strabismus is a visible misalignment of the eyes, with one eye turning in, out, up, down or at an oblique angle.

\section{- Accommodative Esotropia}

Occasionally, when a farsighted child tries to focus to compensate for uncorrected farsightedness, he or she will develop a type of strabismus called accommodative esotropia, where the eyes cross due to excessive focusing effort. This condition usually appears before 2 years of age but also can occur later in childhood. Often, accommodative esotropia can be fully corrected with eyeglasses or contact lenses.

\section{- Esotropia (crossed eyes) needs to be treated early in} life to prevent amblyopia. [5]

Convergence insufficiency can interfere with comfortable reading, causing eye strain, blurred vision, double vision and headaches. One study found that children with exotropia (including convergence insufficiency) at an early age were significantly more likely to develop attention deficit hyperactivity disorder (ADHD), adjustment disorder and learning disabilities by early adulthood. It appears nonsurgical vision therapy can be an effective treatment for convergence insufficiency. This vison impairment could be treated as follows :

-----Eyeglasses or contact lenses - this method may help people who have crossed eyes due to an uncorrected farsightedness.

------Medication (eye drops) - In some cases, as an alternative to patching, eye drops are used in the stronger (good) eye to temporarily blur the vision in the good eye. This forces use of the weaker eye.

--Surgery - straightens and realigns muscles in the eyes; this method has a high success rate although it is expensive and involves more risk than other option

-Patching or covering the better-seeing eye - similar to eye drops, this method works to strengthen the weakened eye.

- Intermittent exotropia $(\mathrm{X}(\mathrm{T}))$ is one of the most common type of strabismus in children as well as adults, especially in the Asian and South Asian populations [6-9]. It is also one of the most common indications for strabismus surgery and its incidence is on the rise. This higher prevalence of exotropia in Asian population compared with West has been attributed to either ethnic differences or to the higher incidence of myopia in the Asian population .

\section{LITERATURE REVIEW FACTORS ASSOCIATED WITH STRABISMUS}

2.1 Social anxiety related conditions and clinical factors [11-12]: clinical factors such as worse diplopia with reduced HRQOL . Other study have shown connection with 
improvement in HRQOL with beliefs, mood and social support .

2.2 Risk Factors : Risk factors for Strabismus include Prematurity ; Amblyopia; Refractive error in children younger than 6 years old; Family history of accommodative esotropia; Family history of infantile esotropia ; Craniofacial disorder; Cranial nerve palsies ; Non-correctable causes of poor vision in one or both eyes ; Thyroid Eye Disease; Genetic Syndromes: Moebius syndrome, Prader-Willi syndrome, mitochondrial myopathies, Down's Syndrome and many others ; Demyelinating or other cerebral disorder; Neuromuscular disorder[10].

2.3 Age factor [13-18]: It has long been debated over timing of surgery with some advocating an early surgery before 4 years of age but was associated with a higher incidence of amblyopia due to the mild overcorrection in the postoperative period in these children with immature visual system. Interestingly, binocular visual integration pathway in these cases is intact, unlike infantile esotropia which warrants early intervention. The exotropia being intermittent, allows for development of binocular fusion and stereopsis. Therefore, delaying the surgery should not influence the sensory and visual outcomes to a great extent, and many studies have advocated for a delayed approach as it may also allow for more accurate measurements and better results.

2.4 Stereopsis, Binocular vision, Amblyopia [19-33]: Distance stereopsis is affected earlier in $\mathrm{X}(\mathrm{T})$ compared with near stereopsis. Some investigators advocate the use of distance stereoacuity to monitor the progression of distance, indication for surgery and as an outcome measure. It is intuitive to consider that better stereopsis must be associated with good control of $\mathrm{X}(\mathrm{T})$ and smaller angle of deviation. However, $\mathrm{X}(\mathrm{T})$ patients can have any possible combination of stereopsis, control and angle of deviation with weak or no correlation between these factors. Similarly, stereopsis or binocular vision has not been found to affect the surgical motor outcomes. Such poor correlation may result from variable measurements of stereopsis and angle of deviation in $\mathrm{X}(\mathrm{T})$ specially in younger children. However, a recent study showed that loss of stereopsis was the only feature associated with poor outcomes on multivariate analysis, which may suggest that if any such association does exist, it may either be affected by multiple factors or too weak to be consistent. On the contrary, surgical correction does improve stereopsis significantly even in the long term .

2.5 Various pre-operative and post-operative factors : Even the surgical dosages used by the surgeons across the world vary which may explain the variability of the reported success rates to some extent. Future studies should further examine these predictive factors to tailor the surgical choice and dosage for optimal results.

\section{LITERARY OBSERVATIONS ABOUT STRABIMSUS AND CONCLUSIONS}

- Strabismus is one of the commonly encountered disease entities in the ophthalmology outpatient department. The prevalence of this disease ranges from $0.5 \%$ to $5 \%$ [10].

- Strabismus, abnormal ocular alignment, is one of the most common ocular problems in children, affecting $5 \%$ of the preschool population [10].

- Strabismus can be treated with conservative therapy such as glasses, prisms, patching and/or orthoptic exercises, with a majority of the cases eventually requiring correction with eye muscle surgery.

- Horizontal eye muscle surgery is performed on patients with exodeviations (out turning), esodeviations (in turning), dissociated vertical deviations, abnormal head postures and/or nystagmus, and is the most common strabismus surgery performed.

- Strabismus can be confirmed with a comprehensive ocular evaluation. A thorough history is obtained regarding; age of onset, symptoms, percentage of time the eye(s) is deviated, whether misalignment occurs when fixating on distance or near objects, other health problems, recent trauma and family history.

- A comprehensive evaluation of a patient with strabismus includes; visual acuity, stereoacuity, 10 prism base down test for ocular preference, measurement of ocular alignment, duction and version movements, slit lamp examination, cycloplegic refraction and fundus ophthalmoscopy.

- The primary goal of strabismus surgery is to improve the alignment of the visual axis of the eyes. Surgery is used to help re-establish binocular single vision (decrease diplopia), increase stereoacuity, improve ocular strain related to intermittent deviations, decrease visual confusion, improve abnormal head posture and restore peripheral visual field [34].

- Strabismus surgery can also help to restore normal facial appearance by straightening the eyes which can have a large socioeconomic impact. In a study performed by Olitsky et al., patients without strabismus had their photos digitally altered to create the appearance of strabismus.

- In infants with congenital esotropia, the success rate of alignment at one year was, $62 \%$ [35]. In those with a re-occurrence of strabismus, repeat surgical intervention can be performed in most cases increasing the percentage of successful realignment. Several large case series report successful surgical realignment rates of $63 \%$ to $81 \%$ in adults, up to one year after surgery [36].

- In adult patients with strabismus, an important determination for post-operative success is the resolution of diplopia. Patients younger than age 10 typically can suppress the deviated eye and therefore don't experience post-operative diplopia. It is important to have clearly defined goals which are agreed upon by the physician and patient prior to surgery.

- While not a true surgical complication, misalignment of the eye post-operatively is the most common cause for patient dissatisfaction. In almost all cases, the post-operative misalignment can be corrected with time, prisms or additional surgery.

- True complications of strabismus surgery occur rarely. Some surgeons instruct patients to stop any blood thinners and anti-coagulants, under the 
supervision of their primary physician, to decrease the risk of intraoperative bleeding.

- There is controversy as to whether this is necessary, except in the case of retrobulbar injections where anticoagulation is a clear risk factor for serious hemorrhage.

- Local anesthetic containing epinephrine can also be used at the time of surgery to help maintain hemostasis, although this is not advocated for peribulbar or subtenon's injections.

- While blood loss during strabismus surgery is minimal, subconjunctival blood looks cosmetically unsatisfactory and can also create increased scarring.

- In most cases, the perforation does not create a problem other than a chorio-retinal scar, but in some cases can trigger endophthalmitis, vitreous hemorrhage or retinal detachment [37]

- Infection rate for strabismus surgery is low and is limited by the use of standard sterile surgical technique [38]. Infection when it occurs, is most commonly seen 2-3 days after surgery.

- Patients may present with redness, pain, discharge and/or lid swelling. Significantly less common but more serious is the risk of the infection spreading into the deeper orbital tissues, creating an orbital cellulitis which requires aggressive antibiotic therapy.

- Some advocate an iris angiogram to ensure adequate anterior segment blood supply in patients with multiple previous strabismus surgeries who are considering undergoing additional strabismus surgery [39].

- Recent advances in imaging techniques and advances in the field of genetics have remarkably changed the recent-day understanding. The advent of high-resolution magnetic resonance imaging (MRI) has led to the discovery of extraocular muscle pulleys which are the condensation of the connective tissue of the posterior Tenon's fascia.

- These help in maintaining the paths of the extraocular muscles. Pulley heterotropia or abnormalities can lead to the development of strabismus. Instability of the rectus pulleys has been shown to be associated with incomitant Strabismus [40].

- Genetic basis of strabismus is also being explored. A recent study by Altick et al.[41] has shown through microarray analysis that the gene expression in the extraocular muscles of the strabismic and the non-strabismic individuals. They found that $25 \%$ of the muscle-specific genes were downregulated in the extraocular muscles of strabismic patients.

\subsection{Amblyopia Management}

- Amblyopia is one of the treatable causes of vision loss in children. Early diagnosis of this condition through visual screening can lead to better treatment outcomes.
- The conventional treatment method involves the occlusion of the better eye. However, the method of occlusion has its very own imperfections. The conventional form of patching has been associated with low compliance, and it averages below 50\% according to a recent study [42].

- It is also associated with social stigma and may lead to stress and anxiety. Recent researches focus on newer treatment modalities to address the issue of low compliance. New liquid crystal display (LCD) occlusion glasses have been developed for amblyopia treatment.

- A study comparing LCD glasses with conventional patching has found the glasses to be an effective alternative [43]. However, they do suffer from the same disadvantage of the spectacle mounted occlusions which can be easily "peeked off" by the children.

\subsection{Imaging in Strabismus}

- Imaging techniques have not only changed the current understanding of Strabismus Etiology but also aided in the planning of the surgery. Anterior chamber optical coherence tomography has been shown to be effective in detecting muscle insertions in previously operated as well as new strabismus cases [44-45].

- Furthermore, they also prove to be more costeffective as compared to CT and MRI. Dynamic MRI[46] is a yet another imaging modality that can help in surgical planning as it can pick up functional muscle contractility. High-resolution MRI of the orbit can successfully predict to extraocular muscle pulley location and the muscle paths which can aid us in planning the surgery.

- The binocular summation is defined as the superiority of binocular visual functions over the monocular ones. Improvement of binocular summation is one of the newly discovered functional benefits of the corrective strabismus surgery [47].

- Minimally Invasive Strabismus Surgery : Minimal invasive approach for strabismus surgery is being explored to decrease tissue trauma and patient discomfort. This surgical technique has been used for various types of strabismus surgeries which include rectus muscle recessions and plications, rectus muscle transpositions, inferior oblique recessions, and rectus muscle posterior fixations [44]. .

- Adjustable Sutures : Adjustable sutures are being used to correct the overcorrections and undercorrections in the immediate postoperative period. These sutures come handy to the Strasbismologist as they give the surgeon an additional chance to increase the overall surgical success rate. Studies have shown better outcomes with adjustable suture surgery than the nonadjustable suture surgery in patients with exotropia [48]. Another study has shown the adjustable suture surgery to be beneficial in children undergoing reoperation for childhood strabismus [49]. Single-stage adjustable strabismus 
surgery is a good alternative for patients with restrictive strabismus [50].

- Psychosocial Aspect of Strabismus [51-56] : Physical appearance is an important aspect of the socialization process. Menon et al. [51] have documented the psychosocial difficulties in individuals with strabismus. Another study has detected subnormal health-related quality of life in the parents of children with strabismus [52]. Strabismus surgery can bring about psychosocial benefits to the affected person [53-54]. Nelson et al. [56] have found strabismus surgery to have a significant effect on self-esteem and confidence of patients. Another study has found that the strabismus surgery improves health-related quality of life in both affected children as well as their parents [57].

\section{ACKNOWLEDGMENTS}

Our thanks to the anonymous reviewers whose comments have helped us in improving the manuscript . Authors are highly obliged and thankful to the team of consultants and staff at Centre for Sight, Delhi for the consultation, help , hospitality and support to conduct the research work.

\section{REFERENCES}

[1] Chia A, Dirani M, Chan Y-H 2010. Prevalence of amblyopia and strabismus in young Singaporean Chinese children. Invest Ophthalmol Vis Sci, 51:34117.doi:10.1167/iovs.09-4461

[2] Bruce A, Santorelli G .2016. Prevalence and risk factors of strabismus in a UK multi-ethnic birth cohort. Strabismus 24:153-160. doi:10.1080/09273972.2016.1242639

[3] CBO, Y., DSP, F., Wong, V.W.Y. 2002. Changing patterns of strabismus: a decade of experience in Hong Kong. Br J Ophthalmology, 86, 854-6.

[4] Mohney, B.G., Huffaker, R.K. 2003. Common forms of childhood exotropia. Ophthalmology, 110:2093-6. doi:10.1016/j.ophtha.2003.04.001

[5] Chew, E., Rernaley, N., Tamboli, A., Jialiang, Z., Podgor, M., Klebanoff, M.1994. Risk factors for esotropia and exotropia. Arch Ophtahlmol. 10, 112.

[6] Chougule, P. Kekunnaya, R. 2017. Surgical management of intermittent exotropia: do we have an answer for all? BMJ journals, BMJ open ophthalmology , 4(1). http://dx.doi.org/10.1136/bmjophth-2018-000243.

[7] Nusz, K.J., Mohney, B.G. 2006. The course of intermittent exotropia in a population-based cohort. Ophthalmology,113:1154-8. doi:10.1016/j.ophtha.2006.01.033.

[8] Hatt, S.R., Gnanaraj, L.2013. Cochrane Eyes and Vision Group . Interventions for intermittent exotropia. Cochrane Database Syst Rev.116. doi:10.1002/14651858.CD003737.pub3

[9] Chiu, A.K.C., Din,N., Ali, N .2014. Standardising reported outcomes of surgery for intermittent exotropia a systematic literature review. Strabismus,22:32-6. doi:10.3109/09273972.2013.877940

[10] Robaei, R., Rose, K.A., Kifley, A., Cosstick, M., Ip J.M., Mitchell, P. 2006. Factors associated with childhood strabismus: findings from a population-based study. Ophthalmol. 7, 113.

[11] Hatt, S.R., Leske, D.A., Liebermann, L., Philbrick, K.L., Holmes, J.M. 2014. Depressive symptoms associated with poor health-related quality of life in adults with strabismus. Ophthalmology, 121(10), 20702071.

[12] McBain, H.B., MacKenzie, K.A., Hancox, J., Ezra, D.G., Adams, G.G., Newman, S.P., 2016. Does strabismus surgery improve quality and mood, and what factors influence this? Eye (Lond). 30(5) , 656-667.

[13] Burke, M.J. 1985. Intermittent exotropia. Int Ophthalmol Clin, 85, 25:53-68. doi:10.1097/00004397-198502540$\underline{00006}$

[14] Richard, J.M. , Parks, M.M 1983. Intermittent exotropia. surgical results in different age groups, Ophthalmology, $90,1172-7$

[15] Stoller, S.H. , Simon, J.W. , Lininger, L.L.1994. Bilateral lateral rectus recession for exotropia: a survival analysis. J Pediatr Ophthalmol Strabismus, 31, 89-92.

[16] Edelman, P.M. , Brown, M.H. , Murphree, A.L. 1988. Consecutive Esodeviation ... then what? Am Orthopt J , $38 \quad$, 111-6. doi:10.1080/0065955X.1988.11981779

[17] Lim, S.H. , Hong, J.S. , Kim, M.M . 2011. Prognostic factors for recurrence with unilateral recess-resect procedure in patients with intermittent exotropia. Eye, 25, 449-54. doi:10.1038/eye.2011.12

[18] Abroms, A.D. , Mohney, B.G. , Rush, D.P. 2001 Timely surgery in intermittent and constant exotropia for superior sensory outcome. Am J Ophthalmol, 131, 1116. doi:10.1016/S0002-9394(00)00623-1

[19] Wang, J. , Hatt, SR.., O'Connor, A.R. 2010. Final version of the distance Randot Stereotest: normative data, reliability, and validity. J Aapos ,14, 142-6. doi:10.1016/j.jaapos.2009.12.159

[20] Holmes, J.M., Birch, E.E., Leske , D.A. 2007. New tests of distance stereoacuity and their role in evaluating intermittent exotropia. Ophthalmology, 114, 1215-20. doi:10.1016/j.ophtha.2006.06.066

[21] Stathacopoulos, R.A. ,Rosenbaum, A.L., Zanoni, D. 1993. Distance stereoacuity. Assessing control in intermittent Exotropia. Ophthalmology,100:495-500.

[22] Yildirim,C. , Mutlu,F.M. ,Chen, Y.1999. Assessment of central and peripheral fusion and near and distance stereoacuity in intermittent exotropic patients before and after strabismus surgery. Am J Ophthalmol ,128, 222-30. doi:10.1016/S0002-9394(99)00079-3

[23] Superstein, R. , Dean, T.W. , Holmes, J.M. 2017 Relationship among clinical factors in childhood intermittent exotropia. J Aapos , 21 , 268-73. doi:10.1016/j.jaapos.2017.04.005

[24] Koklanis , K. , Georgievski, Z. 2009. Recurrence of intermittent exotropia: factors associated with surgical outcomes. Strabismus, doi: $10.1080 / 09273970802678750$

$17: 37-40$.

[25] Zou, D. ,Casafina, C. , Whiteman, A. 2017. Predictors of surgical success in patients with intermittent 
exotropia. J Aapos,

21,

15-18.

doi:10.1016/j.jaapos.2016.11.018

[26] Gezer, A .Sezen, F., Nasri, N. 2004. Factors influencing the outcome of strabismus surgery in patients with exotropia. J Aapos, 8, 56-60. doi:10.1016/j.jaapos.2003.08.006

[27] Hatt S.R., Mohney, B.G. ,Leske, D.A. 2008. Variability of stereoacuity in intermittent exotropia. Am J Ophthalmol, $145 \quad$ 556-61. doi:10.1016/j.ajo.2007.10.028

[28] Adams, W.E. , Leske, D.A., Hatt, S.R.2009. Defining real change in measures of stereoacuity. Ophthalmology, 116:281-5. doi:10.1016/j.ophtha.2008.09.012

[29] Hatt , S.R., Leske, D.A., Liebermann, L. 2012. Variability of angle of deviation measurements in children with intermittent exotropia. J Aapos, 16, 120-4. doi:10.1016/j.jaapos.2011.11.008

[30] Kim, W.J., Kim, M.M . 2017. Variability of preoperative measurements in intermittent exotropia and its effect on surgical outcome. J Aapos, 21, 210-4. doi:10.1016/j.jaapos.2017.05.006

[31] Yang, M. ,Chen, J., Shen, T. $2016 . \quad$ Clinical characteristics and surgical outcomes in patients with intermittent exotropia: a large sample study in South China. Medicine, 95, e2590. doi:10.1097/MD.0000000000002590

[32] Ekdawi, $\quad$ N.S. , Nusz, K.J., Diehl, N.N. 2009. Postoperative outcomes in children with intermittent exotropia from a population-based cohort. J Aapos, 13, 4-7. doi:10.1016/j.jaapos.2008.06.001

[33] Romanchuk, K.G., Dotchin, S.A., Zurevinsky, J. 2006. The natural history of surgically untreated intermittent exotropia-looking into the distant future. J Aapos, 10, 225-31. doi:10.1016/j.jaapos.2006.02.006

[34] Mills, M.D., Coats, D.K., Donahue, S.P., Wheeler, D.T. 2004. American Academy of Ophthalmology. Strabismus surgery for adults: a report by the American Academy of Ophthalmology. Ophthalmol. 6, 111.

[35] Kampanartsanyakorn , S., Surachatkumtonekul, T., Dulayajinda, D., Jumroendararasmee , M., Tongsae, S. 2005. The outcomes of horizontal strabismus surgery and influencing factors of the surgical success. J Med Assoc Thai, 9, 88 .

[36] Beauchamp, G.R., Black, B.C., Coats, DK.., Enzenauer, R.W., Hutchinson, A.K., Saunders, R.A., Simon, J.W., Stager, D.R., Stager, D.R. Jr, Wilson M.E., ZobalRatner, J., Felius, J. 2003. The management of strabismus in adults--I. Clinical characteristics and treatment. J AAPOS. 4,233-40.

[37] Salamon, S.M., Friberg, T.R., Luxenburg, M.N. 1982. Endophthalmitis after strabismus surgery. Am J Ophthalmol 93, 39.

[38] Berger, R.W., Haase , W. 1997. Complications in strabismus surgery. Strabismus, 5, 67.

[39] Saunders, R., Phillips, M.S. 1988. Anterior segment ischemia after three rectus muscle surgery. Ophthalmology, 95, 533.
[40] Von Noorden, G.K., Campos, E.C. Binocular Vision and Ocular Motility: Theory and management of strabismus. Orbis Cyber-

Sight. http://telemedicine.orbis.org/bins/content_page.as p?cid=1-2193-2194

[41] Altick, A.L., Feng, C.Y., Schlauch, K., Johnson, L.A., Von Bartheld, C.S. 2012. Differences in gene expression between strabismic and normal human extraocular muscles. Invest Ophthalmol Vis Sci. 53, 5168-77.

[42] Wallace, M.P., Stewart, C.E., Moseley, M.J., Stephens, D.A., Fielder, A.R. 2013. Monitored Occlusion Treatment Amblyopia Study (MOTAS) Cooperatives; Randomized Occlusion Treatment Amblyopia Study (ROTAS) Cooperatives. Compliance with occlusion therapy for childhood amblyopia. Invest Ophthalmol Vis Sci. 54, 6158-66.

[43] Wang, J., Neely, D.E., Galli, J., Schliesser, J., Graves, A., Damarjian, T.G. 2016.A pilot randomized clinical trial of intermittent occlusion therapy liquid crystal glasses versus traditional patching for treatment of moderate unilateral amblyopia. J AAPOS. 20, 326-31.

[44] Ngo, C.S., Smith, D., Kraft, S.P. 2015. The accuracy of anterior segment optical coherence tomography (ASOCT) in localizing extraocular rectus muscles insertions. J AAPOS. 19, 233-6.

[45] Takkar, B., Sharma, P., Singh, A.K., Sahay, P. 2016. Anterior segment optical coherence tomography for identifying muscle status in strabismus surgery. Int $\mathrm{J}$ Ophthalmol., 9, 933-4.

[46] Teodorescu L, Ionescu, V. 2010. Dynamic nuclear magnetic resonance in strabismus. Oftalmologia. 2010;54:123-8.

[47] Pineles, S.L., Demer, J.L., Isenberg, S.J., Birch, E.E., Velez, F.G. 2015. Improvement in binocular summation after strabismus surgery. JAMA Ophthalmol, 133, 32632.

[48] Mireskandari, K., Cotesta, M., Schofield, J., Kraft, S.P. 2012. Utility of adjustable sutures in primary strabismus surgery and reoperations. Ophthalmology. 119, 629-33.

[49] Zhang, M.S., Hutchinson, A.K., Drack, A.V., Cleveland, J., Lambert, S.R. 2012. Improved ocular alignment with adjustable sutures in adults undergoing strabismus surgery. Ophthalmology.119,396-402.

[50] Sharma, P., Julka, A., Gadia, R., Chhabra, A., Dehran, M. 2009. Evaluation of single-stage adjustable strabismus surgery under conscious sedation. Indian $\mathbf{J}$ Ophthalmol., 57,121-5.

[51] Menon, V., Saha, J., Tandon, R., Mehta, M., Khokhar, S. 2002. Study of the psychosocial aspects of strabismus. J Pediatr Ophthalmol Strabismus. 39, 203-8.

[52] Yamada, T., Hatt, S.R., Leske, D.A., Holmes, J.M. 2011. Health-related quality of life in parents of children with intermittent exotropia. J AAPOS. 15, 135-9.

[53] Glasman, P., Cheeseman, R., Wong, V., Young, J., Durnian, J.M. 2013. Improvement in patients' quality-oflife following strabismus surgery: Evaluation of postoperative outcomes using the Adult Strabismus 20 (AS-20) score. Eye (Lond) , 27, 1249-53. 
[54] Sharma, P.I., Aslam, A., Saxena, R., Vashisht, P. 2017. Responsiveness of Health-Related Quality-of-Life Questionnaire in Adults Undergoing Strabismus Surgery. Presented at AAO Annual Meeting, Chicago.

[55] Nelson, B.A., Gunton, K.B., Lasker, J.N., Nelson, L.B., Drohan, L.A. 2008. The psychosocial aspects of strabismus in teenagers and adults and the impact of surgical correction. J AAPOS. 12, 72-6.e1.

[56] Wang, X., Gao, X., Xiao, M., Tang, L., Wei, X., Zeng, J. 2015. Effectiveness of strabismus surgery on the healthrelated quality of life assessment of children with intermittent exotropia and their parents: A randomized clinical trial. J AAPOS. 19, 298-303. 